\title{
Distribution of generalized mex-related integer partitions
}

\author{
Kalyan Chakraborty and Chiranjit Ray
}

\begin{abstract}
The minimal excludant or "mex" function for an integer partition $\pi$ of a positive integer $n, m e x(\pi)$, is the smallest positive integer that is not a part of $\pi$. Andrews and Newman introduced $\sigma \operatorname{mex}(n)$ to be the sum of $\operatorname{mex}(\pi)$ taken over all partitions $\pi$ of $n$. Ballantine and Merca generalized this combinatorial interpretation to $\sigma_{r} m e x(n)$, as the sum of least $r$-gaps in all partitions of $n$. In this article, we study the arithmetic density of $\sigma_{2} \operatorname{mex}(n)$ and $\sigma_{3} \operatorname{mex}(n)$ modulo $2^{k}$ for any positive integer $k$.
\end{abstract}

Keywords. Minimal excludant, Integer partition; Eta-quotients; Modular forms; Distribution.

2010 Mathematics Subject Classification. Primary: 05A17, 11P83, 11F11, 11F20.

\section{Introduction and statement of results}

In 2015, Fraenkel and Peled [FrPe15] defined the minimal excludant or "mex" function on a set S of positive integers as the least positive integer not in S. Perhaps the notion of the mex function was introduced in the 1930s, and best known for the applications in combinatorics and game theory [Gr39, Sp35].

A partition of a non-negative integer $n$ is a non-increasing sequence of positive integers whose sum is $n$. Let $\pi$ be a partition of $n$ and $\mathcal{P}(n)$ be the set of all partitions of $n$. Recently, Andrews and Newman [AnNe19] considered the minimal excludant function applied to integer partitions. The minimal excludant of $\pi$, denoted mex $(\pi)$, is the smallest positive integer which is not a part of $\pi$. Thus if $\pi$ is $6+4+3+2+1$, a partition of 16 , then $\operatorname{mex}(\pi)=5$. For each positive integer $n$, we have

$$
\operatorname{\sigma mex}(n):=\sum_{\pi \in \mathcal{P}(n)} \operatorname{mex}(\pi) .
$$

For example, $\sigma \operatorname{mex}(4)=9$ with the relevant mex partitions being: $\operatorname{mex}(4)=1, \operatorname{mex}(3+1)=2$, $\operatorname{mex}(2+2)=1, \operatorname{mex}(2+1+1)=3$, and $\operatorname{mex}(1+1+1+1)=2$. The generating function for $\sigma \operatorname{mex}(n)$ is given by

$$
\sum_{n=0}^{\infty} \sigma \operatorname{mex}(n) q^{n}=(-q ; q)_{\infty}^{2},
$$

where the $q$-shifted factorial $(a ; q)_{\infty}:=\prod_{n=1}^{\infty}\left(1-a q^{n-1}\right), \quad|q|<1$. Let $\mathcal{D}_{2}(n)$ be the set of partitions of $n$ into distinct parts using two colors and let $D_{2}(n)=\left|\mathcal{D}_{2}(n)\right|$. In [AnNe19], the authors give two proofs of the following theorem.

Theorem 1.1. Given an integer $n>0$, we have

$$
\operatorname{\sigma mex}(n)=D_{2}(n)
$$

They also studied the parity of $\sigma$ mex function and proved that $\sigma$ mex $(n)$ is almost always even and is odd exactly when $n$ is of the form $j(3 j \pm 1)$, where $j$ is a non-negative integer. 
In some literature, the minimal excludant of a partition $\pi$ is referred to as the least gap or smallest gap of $\pi$. The $r$-gap of a partition $\pi$ is the least positive integer that does not appear at least $r$ times as a part of $\pi$. Let $s_{r}(\pi)$ be the smallest part of the partition $\pi$ appearing less than $r$ times and $S_{r}(n)=\sum_{\pi \in \mathcal{P}(n)} s_{r}(\pi)$.

For example, all the 3 -gaps in the partitions of 5 are: $s_{3}(5)=1, s_{3}(4+1)=1, s_{3}(3+2)=1$, $s_{3}(3+1+1)=1, s_{3}(2+2+1)=1, s_{3}(2+1+1+1)=2, s_{3}(1+1+1+1+1)=2$. Therefore $S_{3}(5)=\sum_{\pi \in \mathcal{P}(5)} s_{3}(\pi)=9$.

Ballantine and Merca [Ba, Ba20] generalized Theorem 1.1 to the sum $S_{r}(n)$ of $r$-gaps in all partitions of $n$. To keep notation uniform, in this article we use $\sigma_{r} \operatorname{mex}(n)$ for $S_{r}(n)$. The generating function for $\sigma_{r} \operatorname{mex}(n)$ is given by

$$
\sum_{n=0}^{\infty} \sigma_{r} \operatorname{mex}(n) q^{n}=\frac{\left(q^{2 r} ; q^{2 r}\right)_{\infty}}{(q ; q)_{\infty}\left(q^{r} ; q^{2 r}\right)_{\infty}} .
$$

In [Ba20], Ballantine and Merca proved the following identity.

Theorem 1.2. For $n \geq 0$ and $r \geq 1$ we have

$$
\sum_{k=0}^{\infty} p\left(n-r T_{k}\right)=\sigma_{r} \operatorname{mex}(n),
$$

where $p(n)$ counts the number of partition of $n$ and $T_{k}$ is the $k$-th triangular number.

Recently, Ray and Barman [RaBa20, Theorem 1.6], studied the divisibility of Uncu's partition function $\mathcal{E O}_{u}(n)$. After some elementary calculations we observe that the generating function of $\mathcal{E O}_{u}(2 n)$ and $\sigma \operatorname{mex}(n)$ (or $\left.\sigma_{1} \operatorname{mex}(n)\right)$ are the same. So using the result mentioned above, $\sigma \operatorname{mex}(n)$ is almost always divisible by $2^{k}$ for any positive integer $k$.

A well-known conjecture of Parkin and Shanks [PS67], for integer partitions $p(n)$, states that the even and odd values of $p(n)$ are equally distributed, that is,

$$
\lim _{X \rightarrow \infty} \frac{\#\{0 \leq n \leq X: p(n) \equiv a \quad(\bmod 2)\}}{X}=\frac{1}{2},
$$

where $a \in\{0,1\}$. Little is known regarding this conjecture. In the following theorem we prove that $\sigma_{2} \operatorname{mex}(n)$ and $\sigma_{3} \operatorname{mex}(n)$ are almost always even. More generally we prove the following result.

Theorem 1.3. (Main Theorem) Let $k$ be a positive integer and $r \in\{2,3\}$. Then

$$
\lim _{X \rightarrow+\infty} \frac{\#\left\{0 \leq n<X: \sigma_{r} \operatorname{mex}(n) \equiv 0\left(\bmod 2^{k}\right)\right\}}{X}=1 .
$$

In other words for almost every non-negative integer $n$ lying in an arithmetic progression, the integer $\sigma_{r} \operatorname{mex}(n)$ is a multiple of $2^{k}$ where $r \in\{2,3\}$.

\section{Preliminaries}

In this section, we recall some definitions and facts relating to the arithmetic of classical modular forms. For more details, one can consult [On04, Ko93]. Let $\mathbb{H}$ denote the upper-half plane. The complex vector space of modular forms of weight $\ell$ (a positive integer) with respect to a congruence subgroup $\Gamma$ will be denoted by $M_{\ell}(\Gamma)$. 
Definition 2.1. [On04, Definition 1.15] Let $\chi$ be a Dirichlet character modulo $N$ (a positive integer). Then a modular form $f \in M_{\ell}\left(\Gamma_{1}(N)\right)$ has Nebentypus character $\chi$ if

$$
f\left(\frac{a z+b}{c z+d}\right)=\chi(d)(c z+d)^{\ell} f(z)
$$

for all $z \in \mathbb{H}$ and all $\left[\begin{array}{ll}a & b \\ c & d\end{array}\right] \in \Gamma_{0}(N)$. The space of such modular forms is denoted by $M_{\ell}\left(\Gamma_{0}(N), \chi\right)$. Here $\Gamma_{0}(N)$ will be as usual the Hecke congruence subgroup of level $N$.

Recall that Dedekind's eta-function is defined by

$$
\eta(z):=q^{1 / 24}(q ; q)_{\infty}=q^{1 / 24} \prod_{n=1}^{\infty}\left(1-q^{n}\right),
$$

where $q=e^{2 \pi i z}$ and $z \in \mathbb{H}$. A function $f(z)$ is called an eta-quotient if it is of the form

$$
f(z)=\prod_{\delta \mid N} \eta(\delta z)^{r_{\delta}},
$$

where $N$ is a positive integer and $r_{\delta}$ is an integer.

We now recall two theorems from [On04, p. 18], which help us check the modularity of eta-quotients that show up in our study.

Theorem 2.2. [On04, Theorem 1.64] Suppose that $f(z)=\prod_{\delta \mid N} \eta(\delta z)^{r_{\delta}}$ is an eta-quotient such that

$$
\begin{aligned}
\ell & =\frac{1}{2} \sum_{\delta \mid N} r_{\delta} \in \mathbb{Z} \\
\sum_{\delta \mid N} \frac{N}{\delta} r_{\delta} & \equiv 0(\bmod 24) .
\end{aligned}
$$

Then

$$
f\left(\frac{a z+b}{c z+d}\right)=\chi(d)(c z+d)^{\ell} f(z)
$$

for every $\left[\begin{array}{ll}a & b \\ c & d\end{array}\right] \in \Gamma_{0}(N)$. Here

$$
\chi(d):=\left(\frac{(-1)^{\ell} \prod_{\delta \mid N} \delta^{r_{\delta}}}{d}\right) .
$$

Suppose that $f$ is an eta-quotient satisfying the conditions of Theorem 2.2. If $f$ is also holomorphic at all of the cusps of $\Gamma_{0}(N)$, then $f \in M_{\ell}\left(\Gamma_{0}(N), \chi\right)$. To check the holomorphicity at cusps of $f(z)$ it suffices to check that the orders at the cusps are non-negative. The necessary criterion for determining orders of an eta-quotient at cusps is the following.

Theorem 2.3. [On04, Theorem 1.65] Let $c, d$, and $N$ be positive integers with $d \mid N$ and $\operatorname{gcd}(c, d)=$ 1. If $f(z)$ is an eta-quotient satisfying the conditions of Theorem 2.2 for $N$, then the order of vanishing of $f(z)$ at the cusp $\frac{c}{d}$ is

$$
\frac{N}{24} \sum_{\delta \mid N} \frac{\operatorname{gcd}(d, \delta)^{2} r_{\delta}}{\operatorname{gcd}\left(d, \frac{N}{d}\right) d \delta}
$$




\section{Proof of main results}

In this section, we prove Theorem 1.3. We prove the following lemmas.

Lemma 3.1. Let $k$ be a positive integer and $r \in\{2,3\}$. Then

$$
\frac{\eta(24 r z)^{2^{k}-1}}{\eta(24 z) \eta(48 r z)^{2^{k-1}-2}} \equiv \sum_{n=0}^{\infty} \sigma_{r} \operatorname{mex}(n) q^{24 n+3 r-1}\left(\bmod 2^{k}\right) .
$$

Proof. From (1.1), the generating function of $\sigma_{r} \operatorname{mex}(n)$ is given by

$$
\sum_{n=0}^{\infty} \sigma_{r} \operatorname{mex}(n) q^{n}=\frac{\left(q^{2 r} ; q^{2 r}\right)_{\infty}^{2}}{(q ; q)_{\infty}\left(q^{r} ; q^{r}\right)_{\infty}}
$$

Consider

$$
\mathcal{A}(z)=\prod_{n=1}^{\infty} \frac{\left(1-q^{24 r n}\right)^{2}}{\left(1-q^{48 r n}\right)}=\frac{\eta(24 r z)^{2}}{\eta(48 r z)} .
$$

By the binomial theorem, for any positive integers $r$ and $k$ we have

$$
\left(q^{r} ; q^{r}\right)_{\infty}^{2^{k}} \equiv\left(q^{2 r} ; q^{2 r}\right)_{\infty}^{2^{k-1}}\left(\bmod 2^{k}\right)
$$

Therefore,

$$
\mathcal{A}^{2^{k-1}}(z)=\frac{\eta(24 r z)^{2^{k}}}{\eta(48 r z)^{2^{k-1}}} \equiv 1\left(\bmod 2^{k}\right)
$$

Define $\mathcal{B}_{r, k}(z)$ by

$$
\mathcal{B}_{r, k}(z)=\frac{\eta(48 r z)^{2}}{\eta(24 z) \eta(24 r z)} \mathcal{A}^{2^{k-1}}(z)
$$

Now, modulo $2^{k}$, we have

$$
\begin{aligned}
\mathcal{B}_{r, k}(z) & =\frac{\eta(48 r z)^{2}}{\eta(24 z) \eta(24 r z)} \frac{\eta(24 r z)^{2^{k}}}{\eta(48 r z)^{2^{k-1}}} \\
& \equiv \frac{\eta(48 r z)^{2}}{\eta(24 z) \eta(24 r z)} \\
& =q^{3 r-1} \frac{\left(q^{48 r} ; q^{48 r}\right)_{\infty}^{2}}{\left(q^{24} ; q^{24}\right)_{\infty}\left(q^{24 r} ; q^{24 r}\right)_{\infty}} .
\end{aligned}
$$

Since

$$
\mathcal{B}_{r, k}(z)=\frac{\eta(24 r z)^{2^{k}-1}}{\eta(24 z) \eta(48 r z)^{2^{k-1}-2}},
$$

combining (3.2) and (3.3), we obtain the required result.

Lemma 3.2. Let $k>1$ be a positive integer and $r \in\{2,3\}$. Then

$$
\mathcal{B}_{r, k}(z)=\frac{\eta(24 r z)^{2^{k}-1}}{\eta(24 z) \eta(48 r z)^{2^{k-1}-2}} \in M_{2^{k-2}}\left(\Gamma_{0}(L), \chi(\bullet)\right),
$$

where

$$
L= \begin{cases}1152 & \text { if } r=2 \\ 576 & \text { if } r=3\end{cases}
$$


Proof. First, we use Theorem 2.2 and find the following:

1. The weight of the eta-quotient $\mathcal{B}_{r, k}(z)$ is $2^{k-2}$.

2. Suppose the level of the eta-quotient $\mathcal{B}_{r, k}(z)$ is $48 \mathrm{ru}$, where $u$ is the smallest positive integer satisfying the following identity.

$$
\frac{48 r u}{24 r}\left(2^{k}-1\right)-\frac{48 r u}{24}-\frac{48 r u}{48 r}\left(2^{k-1}-2\right) \equiv 0(\bmod 24)
$$

Equivalently, we have

$$
u\left(3 \cdot 2^{k-1}-2 r\right) \equiv 0(\bmod 24) .
$$

Since $k>1$, we have $u=12$ if $r=2$ and $u=4$ if $r=3$. Hence level of the eta-quotient $\mathcal{B}_{r, k}(z)$ is

$$
\begin{cases}1152 & \text { if } r=2 \\ 576 & \text { if } r=3\end{cases}
$$

3. The Nebentypus character is

$$
\chi(\bullet)=\left(\frac{(-1)^{2^{k-2}}(24 r)^{2^{k}-1}(24)^{-1}(48 r)^{-2^{k-1}+2}}{\bullet}\right) .
$$

By Theorem 2.3, the cusps of $\Gamma_{0}(L)$ are given by $\frac{c}{d}$ where $d \mid L$ and $\operatorname{gcd}(c, d)=1$. Now note that eta-quotient $\mathcal{B}_{r, k}(z)$ is holomorphic at a cusp $\frac{c}{d}$ if and only if

$$
\left(2^{k}-1\right) \frac{\operatorname{gcd}(d, 24 r)^{2}}{24 r}-\frac{\operatorname{gcd}(d, 24)^{2}}{24}-\left(2^{k-1}-2\right) \frac{\operatorname{gcd}(d, 48 r)^{2}}{48 r} \geq 0 .
$$

Equivalently, if and only if

$$
2\left(2^{k}-1\right) \frac{\operatorname{gcd}(d, 24 r)^{2}}{\operatorname{gcd}(d, 48 r)^{2}}-2 r \frac{\operatorname{gcd}(d, 24)^{2}}{\operatorname{gcd}(d, 48 r)^{2}}-\left(2^{k-1}-2\right) \geq 0 .
$$

Case (i). When $r=2$ then the left side of (3.4) can be written as

$$
2\left(2^{k}-1\right) \frac{\operatorname{gcd}(d, 48)^{2}}{\operatorname{gcd}(d, 96)^{2}}-4 \frac{\operatorname{gcd}(d, 24)^{2}}{\operatorname{gcd}(d, 96)^{2}}-\left(2^{k-1}-2\right) \geq 0 .
$$

To check the positivity of (3.5), we have to find all the possible divisors of 1152 . We define three sets as follows

$$
\begin{aligned}
& \mathcal{H}_{1}=\left\{2^{\alpha} 3^{\beta}: 0 \leq \alpha \leq 3,0 \leq \beta \leq 2\right\}, \\
& \mathcal{H}_{2}=\left\{2^{\alpha} 3^{\beta}: \alpha=4,0 \leq \beta \leq 2\right\}, \\
& \mathcal{H}_{3}=\left\{2^{\alpha} 3^{\beta}: 5 \leq \alpha \leq 7,0 \leq \beta \leq 2\right\} .
\end{aligned}
$$

Note that $\mathcal{H}_{1} \cup \mathcal{H}_{2} \cup \mathcal{H}_{3}$ contains all positive divisors of 1152. In the following table we compute all necessary data to prove the positivity of (3.5). 


\begin{tabular}{|l|c|c|c|}
\hline $\begin{array}{l}\text { Values of } d \text { such } \\
\text { that } d \mid 1152\end{array}$ & $\frac{\operatorname{gcd}(d, 48)^{2}}{\operatorname{gcd}(d, 96)^{2}}$ & $\frac{\operatorname{gcd}(d, 24)^{2}}{\operatorname{gcd}(d, 96)^{2}}$ & Values of $(3.5)$ \\
\hline$d \in \mathcal{H}_{1}$ & 1 & 1 & $2^{k-1} 3-4$ \\
\hline$d \in \mathcal{H}_{2}$ & 1 & $1 / 4$ & $2^{k-1} 3-1$ \\
\hline$d \in \mathcal{H}_{3}$ & $1 / 4$ & $1 / 16$ & $5 / 4$ \\
\hline
\end{tabular}

Since $k>1$, it is clear from the above table that the quantities in (3.5) are always greater than equal to 0 for any positive integer $d \mid 1152$.

Case (ii). When $r=3$ then the left side of (3.4) can be written as

$$
2\left(2^{k}-1\right) \frac{\operatorname{gcd}(d, 72)^{2}}{\operatorname{gcd}(d, 144)^{2}}-6 \frac{\operatorname{gcd}(d, 24)^{2}}{\operatorname{gcd}(d, 144)^{2}}-\left(2^{k-1}-2\right) \geq 0 .
$$

To check the positivity of (3.6), we have to find all the possible divisors of 576 . We define four sets as follows

$$
\begin{array}{ll}
\mathcal{G}_{1}=\left\{2^{\alpha} 3^{\beta}: 0 \leq \alpha \leq 3,0 \leq \beta \leq 1\right\}, & \mathcal{G}_{2}=\left\{2^{\alpha} 3^{\beta}: 0 \leq \alpha \leq 3, \beta=2\right\}, \\
\mathcal{G}_{3}=\left\{2^{\alpha} 3^{\beta}: 4 \leq \alpha \leq 6,0 \leq \beta \leq 1\right\}, & \mathcal{G}_{4}=\left\{2^{\alpha} 3^{\beta}: 4 \leq \alpha \leq 6, \beta=2\right\} .
\end{array}
$$

Note that $\mathcal{G}_{1} \cup \mathcal{G}_{2} \cup \mathcal{G}_{3} \cup \mathcal{G}_{4}$ contains all positive divisors of 576. In the following table we compute all necessary data to prove the positivity of (3.6).

\begin{tabular}{|l|c|c|c|}
\hline $\begin{array}{l}\text { Values of } d \text { such } \\
\text { that } d \mid 576\end{array}$ & $\frac{\operatorname{gcd}(d, 72)^{2}}{\operatorname{gcd}(d, 144)^{2}}$ & $\frac{\operatorname{gcd}(d, 24)^{2}}{\operatorname{gcd}(d, 144)^{2}}$ & Values of (3.6) \\
\hline$d \in \mathcal{G}_{1}$ & 1 & 1 & $2^{k-1} 3-6$ \\
\hline$d \in \mathcal{G}_{2}$ & 1 & $1 / 9$ & $2^{k-1} 3-2 / 3$ \\
\hline$d \in \mathcal{G}_{3}$ & $1 / 4$ & $1 / 4$ & 0 \\
\hline$d \in \mathcal{G}_{4}$ & $1 / 4$ & $1 / 36$ & $4 / 3$ \\
\hline
\end{tabular}

Since $k>1$, it is clear from the above table that the quantities in (3.6) are always greater than equal to 0 for any positive integer $d \mid 576$.

Therefore, by Case (i) and Case (ii), the eta-quotient $\mathcal{B}_{r, k}(z)$, where $r \in\{2,3\}$ and $k>1$, are holomorphic at every cusp $\frac{c}{d}$ and hence it is a modular form on $\Gamma_{0}(L)$ with Nebentypus character $\chi(\bullet)$. This completes the proof of Lemma 3.2.

We state the following result of Serre.

Theorem 3.3. [On04, Theorem 2.65] Let $k, m$ be positive integers. If $f(z) \in M_{k}\left(\Gamma_{0}(N), \chi(\bullet)\right)$ has the Fourier expansion $f(z)=\sum_{n=0}^{\infty} c(n) q^{n} \in \mathbb{Z}[[q]]$, then there is a constant $\alpha>0$ such that

$$
\#\{n \leq X: c(n) \not \equiv 0(\bmod m)\}=\mathcal{O}\left(\frac{X}{\log ^{\alpha} X}\right) .
$$

Proof of Theorem 1.3. Suppose $k>1$ is a positive integer and $r \in\{2,3\}$. From Lemma 3.2, we have

$$
\mathcal{B}_{r, k}(z)=\frac{\eta(24 r z)^{2^{k}-1}}{\eta(24 z) \eta(48 r z)^{2^{k-1}-2}} \in M_{2^{k-2}}\left(\Gamma_{0}(L), \chi(\bullet)\right) .
$$


Also the Fourier coefficients of the eta-quotient $\mathcal{B}_{r, k}(z)$ are integers. So, by Theorem 3.3 and Lemma 3.1 , we can find a constant $\alpha>0$ such that

$$
\#\left\{n \leq X: \sigma_{r} \operatorname{mex}(n) \not \equiv 0\left(\bmod 2^{k}\right)\right\}=\mathcal{O}\left(\frac{X}{\log ^{\alpha} X}\right) .
$$

Hence

$$
\lim _{X \rightarrow+\infty} \frac{\#\left\{n \leq X: \sigma_{r} \operatorname{mex}(n) \equiv 0\left(\bmod 2^{k}\right)\right\}}{X}=1 .
$$

This completes the proof of Theorem 1.3.

Acknowledgement. The second author has carried out this work at Harish-Chandra Research Institute, affiliated with Homi Bhabha National Institute (Department of Atomic Energy, India), as a Postdoctoral Fellow. We thank the anonymous referee for his/her thorough review and highly appreciate the comments and suggestions, which significantly contributed to improve this article.

\section{References}

[AnNe19] Andrews, G.E. and Newman, D., Partitions and the minimal excludant, Ann. Comb., 23, (2019), 249-254.

[Ba20] Ballantine, C. and Merca, M., Bisected theta series, least $r$-gaps in partitions, and polygonal numbers, Ramanujan J., 52, (2020), 433-444.

[Ba] Ballantine, C. and Merca, M., Combinatorial Proof of the Minimal Excludant Theorem, Arxiv, (arXiv:1908.06789).

[FrPe15] Fraenkel, A.S. and Peled, U., Harnessing the unwieldy MEX function, Games of no chance 4, volume 63 of Math. Sci. Res. Inst. Publ., 77-94. Cambridge Univ. Press, New York, 2015.

[Gr39] Grundy, P. M., Mathematics and games, Eureka, 2, (1939), 6-9.

[Ko93] Koblitz, N., Introduction to elliptic curves and modular forms, volume 97 of Graduate Texts in Mathematics, SpringerVerlag, New York, second edition, 1993.

[On04] Ono, K., The web of modularity: arithmetic of the coefficients of modular forms and q-series, volume 102 of CBMS Regional Conference Series in Mathematics. Published for the Conference Board of the Mathematical Sciences, Washington, DC; by the American Mathematical Society, Providence, RI, 2004.

[PS67] Parkin, T.R. and Shanks, D., On the distribution of parity in the partition function, Math. Comp., 21, (1967), 466-480.

[RaBa20] Ray, C. and Barman, R., On Andrews' integer partitions with even parts below odd parts, J. Number Theory, 215, (2020), 321-338.

[Sp35] R. Sprague, Über mathematische Kampfspiele, Tohoku Mathematical Journal, 41 (1935), $438-444$.

\section{Kalyan Chakraborty}

Department of Mathematics

Kerala School of Mathematics

Kozhikode - 673571

Kerala, INDIA

e-mail:director@ksom.res.in

\section{Chiranjit Ray}

Department of Mathematics

Harish-Chandra Research Institute

Prayagraj - 211019

Uttar Pradesh, INDIA

e-mail: chiranjitray.m@gmail.com 